Questions vives

\section{Questions Vives}

Recherches en éducation

$\mathbf{N}^{\circ} 34 \mid 2020$

Expériences vécues et surgissement d'événements : une écriture du sensible en recherche biographique

\title{
Expérience sensible du corps de chair et phénoménologie de l'événement : vers une éthique de la délicatesse
}

\section{Martine Janner Raimondi}

\section{CpenEdition}

Édition électronique

URL : https://journals.openedition.org/questionsvives/5483

DOI : 10.4000/questionsvives.5483

ISSN : 1775-433X

Éditeur

Université Aix-Marseille (AMU)

Édition imprimée

Date de publication : 18 décembre 2020

ISBN : 978-2-912643-58-2

ISSN : 1635-4079

Référence électronique

Martine Janner Raimondi, «Expérience sensible du corps de chair et phénoménologie de l'événement : vers une éthique de la délicatesse », Questions Vives [En ligne], №34 | 2020, mis en ligne le 18 décembre 2020, consulté le 11 octobre 2021. URL : http://journals.openedition.org/ questionsvives/5483; DOI : https://doi.org/10.4000/questionsvives.5483

Ce document a été généré automatiquement le 11 octobre 2021.

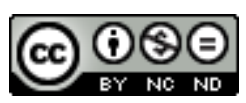

Questions Vives est mis à disposition selon les termes de la licence Creative Commons Attribution Pas d'Utilisation Commerciale - Pas de Modification 4.0 International. 


\title{
Expérience sensible du corps de chair et phénoménologie de l'événement : vers une éthique de la délicatesse
}

\author{
Martine Janner Raimondi
}

1 Lorsqu'en recherche, l'objectif consiste à prendre en compte les expériences vécues aux tréfonds d'un corps de chair, rapidement la dimension sensible des éprouvés et des apprentissages en lien avec une intelligence pratique en lien avec des événements situés dans le temps, un lieu, un contexte et un territoire, émerge. Revisitant à nouveaux frais le constat de Rancière (2000) concernant le partage du sensible, c'est-àdire «la répartition des parts et des places» fondée «sur un partage des espaces, des temps et des formes d'activité » déterminant « comment les uns et les autres ont part à ce partage » (Ibid., p. 7), nous questionnons les logiques, démarches et configurations $\mathrm{du}$ l'ordre du dicible et du visible en recherche, qui répartissent ce qu'il est possible d'entendre comme discours. En sciences humaines et sociales, particulièrement, $a$ fortiori quand il s'agit de porter attention aux expériences vécues et aux apprentissages pouvant en découler, comme c'est le cas en recherche biographique en éducation, il importe d'interroger la dimension et le partage du sensible du point de vue de la recherche, précisément parce que les visées d'objectivation réclament une légitimité académique, qui tend à évacuer la subjectivité. Or, la dimension sensible transparaît autant du côté des chercheurs, que de celui des personnes, premières concernées par une étude empirique. En effet, les productions scientifiques ne peuvent occulter ni les éléments sensibles des expériences humaines dans le monde commun de la vie, ni les résonnances tout à la fois cognitives et sensibles, chez le chercheur et les personnes humaines lors des rencontres organisées dans le cadre d'une enquête, faisant surgir l'autre en soi et le soi dans l'autre.

2 Dans les entrelacs entre sensibilité et rationalité, la subtilité et la complexité des croisements est telle parfois, qu'il est parfois difficile d'en démêler les nouages. 
Considérer l'expérience sensible d'un sujet humain, lui accorder de la valeur, chercher à l'appréhender, puis d'en rendre compte, nous a permis de comprendre combien l'approche phénoménologique est requise au départ, précisément parce qu'elle permet de la rendre visible le phénomène de l'expérience vécue, éprouvée subjectivement. Toutefois, les restitutions et analyses compréhensives effectuées en recherche nécessitent un travail herméneutique d'interprétation qui vient compléter l'approche phénoménologique, préalable et première qui en resterait au seul niveau de la description des phénomènes.

Dans la mesure où nous nous intéressons aux dimensions biographiques des parcours vie des personnes humaines, il nous a semblé important d'interroger le processus de construction de sens de cette expérience en lien avec la notion d'événement. Pour ce faire, nous nous appuierons notamment sur deux ouvrages de Romano, L'événement et le monde, publié en 1998 et Au cœur de la raison: la phénoménologie (2010). Ensuite, nous ouvrirons la question aux dimensions éthiques, méthodologiques et politiques de prise en compte de l'expérience sensible, en posant la question de la posture de recherche, avant de présenter la structure et les articles qui composent ce numéro 34 de la revue.

\section{De l'expérience vécue à l'événement de sens}

4 Comment appréhender et rendre compte des expériences sensibles en recherche ? Telle est la première question de recherche qui traverse ce numéro. Cette réflexion sur la prise en compte du sensible dans le domaine de la raison, rappelle la « raison au grand cœur " de Romano $(2010$, p. 14) en écho à sa notion d'événementialité qui permet d'ouvrir autant à l'infra logique du sensible, qu'aux différentes constructions de sens produites, non sans résonnance avec des éléments du contexte historico-culturel et sociétal. Par-delà les enjeux épistémologiques, il s'agit de repérer humblement les tenants d'une éthique de la délicatesse propre à endiguer le manque de respect.

Pour Romano (1998), l'événement est ce qui advient à un sujet humain et dont le sens reconfigure son rapport au monde. "L'événement signifie, pour l'advenant [le sujet à qui advient l'événement], l'avènement d'un nouveau monde » (Ibid., p. 55). La notion d'événement ne relève d'aucune relation causale, en cela, nous pourrions établir un lien avec la contingence posée par Husserl et reprise ensuite par Ricœur. L'événement, qui ne peut être donc réduit à un fait uniquement pris dans une relation de causalité, surgit non de nulle part, mais survient de façon impromptue, comme par effraction. Lorsque nous vivons l'expérience de la naissance, de la mort d'un proche, d'un accident ou d'une maladie, n'avons-nous pas en effet cette impression première de vivre ces événements comme s'ils surgissaient de façon abrupte et imprévisible, venant ainsi bouleverser notre rapport au monde initial mais également notre rapport aux autres et à nous-mêmes? Le sens de l'événement considéré sous l'angle de la phénoménologie déborde ce qui peut en être dit, notamment au plan rationnel, comme le précise Romano (1998) :

Certes, l'événement se produit bien aussi comme fait, et à ce titre, il a des causes ; mais il n'est événement que parce qu'il transcende ses causes dont l'enchaînement se produit toujours nécessairement dans l'horizon du monde, compris comme 'horizon de possibles préexistants. De l'événement, par conséquent, on ne peut dire qu'une chose : il arrive " parce qu'il arrive » (...) L'événement est « sans pourquoi, il se soustrait, par principe, au principe de raison : (Ibid., p. 80). 
Cette considération de l'événement est assortie à une distinction que Romano (1998) reprend d'Eschyle, comme le précise Cabestan (2016), entre l'ex-pér-ience en tant qu'épreuve, lorsqu'il parle de la "connaissance par l'épreuve (to pathei mathos) et l'expérience au sens empirique du terme (Cabestan, 2016, p.9, note 7). Le premier terme "ex-pér-ience " s'ouvre à l'événement dans ce qu'il apporte et révèle comme potentialités, y compris en termes d'apprentissage sur soi, sur le monde et les autres; alors que le second renvoie à la facticité du phénomène et la logique rationnelle dans laquelle il s'insère lorsqu'il est mis en corrélation avec les attendus scientifiques objectivant. En épistémologie, ces derniers sont caractérisés par la reproductibilité et l'identification des conditions d'émergence visant à la prédictibilité du phénomène étudié.

\section{Un événemential qui ouvre sur un au-delà des faits}

7 Romano (1998) distingue ainsi le concept événemential du monde, de celui événementiel du monde. L'événemential concerne «la totalité articulée de possibilités à partir de laquelle l'advenant [le sujet auquel advient l'événément] se détermine luimême en son aventure" (Ibid., p.91). L'événementiel renvoie à "l'essaim de circonstances qui éclairent le fait lui-même sans pour autant en faire partie " (Ibid., p. 91). Nous comprenons que tout phénomène qui apparaît, peut être ainsi considéré sous deux angles différents: soit, sous l'angle événemential; soit, sous l'angle événementiel. Cela dépend du type de regard porté et des enjeux épistémologiques visés. Vallée (2016) rapproche cette distinction de celle établie par Aristote, dans la Métaphysique, livre A, entre constater et connaître, entre "savoir que " et, "savoir pourquoi »:

Ainsi, quelqu'un peut tomber malade et savoir qu'il est malade, sans trop savoir pourquoi il en est ainsi. Et, il peut aussi s'enquérir des causes de sa maladie, consulter un médecin, etc., en tenant pour acquis que cette maladie a été causée par quelque chose. (...) Sur la base de cette distinction (...), Aristote propose une distinction entre deux types d'homme : soit les hommes d'expérience, qui savent quelque chose tout en ignorant les raisons et les causes, et les hommes d'art, qui eux connaissent le pourquoi des choses. Or, il est manifeste, pour Aristote, que la connaissance des causes et des raisons est supérieure à la simple constatation ou au savoir que..., puisqu'il s'agit d'une compréhension plus complète des choses, même si dans le contexte de la vie pratique, l'homme d'expérience peut à l'occasion se montrer plus habile et plus avisé que l'homme d'art : (Ibid., p. 23).

La question se pose de savoir si l'approche phénoménologique événementiale apporterait un savoir de moindre valeur épistémologique? Deux arguments plaident au contraire en faveur de sa légitime prise en compte. Le premier, en accord avec l'argumentation déployée par Vallée (2016) et Romano (1998), rappelle combien l'exigence même de la phénoménologie consiste à ne pas oublier la dimension événementiale de l'expérience, c'est-à-dire : cette dimension inaugurale de l'expérience qui ouvre à nouveaux frais le champ des possibles, contrairement à l'approche par la raison qui rabat l'événementialité de l'expérience à ce qui peut être mis en relation sous forme de répétabilité et de prédictibilité. Ce premier argument renvoie à une approche qui considère un savoir que à propos du phénomène considéré, non sous la forme d'un raisonnement logique-mathématique, mais sous la forme d'une appréhension en lien avec des ressemblances, des dissemblances, des constances ou non, venant réinterroger le sens de ce qui arrive par-delà le raisonnement logique sur ce qui est possible ou non, 
souhaitable ou non. Vallée (2016) formule ce que vise une phénoménologie herméneutique de l'événement en ces termes: «elle cherche à décrire ce qui caractérise l'événement, par rapport à d'autres types de phénomènes comme un objet perçu ou la représentation d'un être imaginaire » (ibid., p. 26).

Un second argument peut être ajouté, en relation avec l'enjeu épistémologique de construction de connaissances propre à la recherche. En effet, se saisir de l'événementialité d'une expérience du monde faite par un sujet humain, ne conduit-il pas : d'une part, à commencer par porter attention à ce "donné » de l'expérience sensible propre, notamment en maintenant ouverte sa pensée de l'expérience ; d'autre part, à se saisir de ces premiers rapports analogiques de ressemblance, de dissemblance, de constance ou non? D'où viendraient nos compétences, les apprentissages issus de nos expériences sensibles du monde, nos ruses pratiques - nos métis -en lien avec notre intelligence pratique, nos « arts de faire ", si ce n'était sur fond d'expériences sensibles incarnées? Dit autrement, loin de considérer le « savoir que ", comme un savoir de moindre valeur réservé à l'homme commun, considérons avec Romano $(1998,2010)$ et Marion (2010) dans le sillage de la pensée de Husserl, que le savoir d'ex-pér-ience possède une valeur propre, qui contribue à compléter la connaissance rationnelle. Il y a là, comme une démocratisation du savoir dans le sens où le savoir de l'homme d'ex-pér-ience vaut également même si ce n'est pas au même titre que la connaissance de l'homme d'art, de l'expert ou du savant. Si l'un comme l'autre ont une valeur, cette valeur ne renvoie ni aux mêmes modalités d'élaboration de savoir, ni aux mêmes contenus de savoir, ni encore aux mêmes enjeux de vie. En effet, dans le savoir que, la dimension événementiale de l'expérience sensible du monde comporte outre, un apprentissage tel qu'il a été précisé, un questionnement sur son être propre, sur le soi, sa vie, ses proches et le monde. Car, cette "ex-pér-ience" contribue à la formation - au sens de Bildung - de l'homme lui-même et des autres, pour peu que ce savoir puisse être dit, écrit, lu, partagé avec d'autres et reconnu. C'est précisément l'un des buts poursuivis par Delory-Momberger (2014, 2009), dans son élaboration théorique de la recherche biographique.

\section{L'événement, une expérience sensible instauratrice de monde}

Pour Vallée (2016), la nouveauté de la phénoménologie herméneutique de Romano réside dans :

(...) la thèse forte selon laquelle tout événement, voire tout phénomène pour autant qu'il se donne ou survienne, est par lui-même origine et porteur de son sens. Le sens d'un événement ne se laisserait comprendre qu'à partir de l'événement luimême, et non à partir d'un éclairage venant des causes ou des raisons qu'on pourrait lui attribuer: (Ibid., p. 28).

11 L'importance accordée à l'événement comprenant son sens, invite à considérer la question de la posture du sujet humain - l'advenant - face à lui. Car il s'agit bien de se préparer à accueillir l'événement comme phénomène expérientiel sensible afin de permettre à l'advenant de le comprendre et par là-même de se comprendre. Nous repérons là un enjeu d'un autre ordre, celui d'un processus de formation qui s'y trouve d'emblée impliqué, à savoir un processus de subjectivation renouvelé, ouvrant un champ de possibles. Dans la pensée de Maldiney (1991): «l'événement est jet du monde » (Ibid., p. 284), « un transpossible auquel le soi a ouverture» (Ibid., p. 143). Mais comment définir l'événement? Romano (1998) répond: 
'Evénement' au sens " propre », puisque, étymologiquement « événement » vient du latin evenire, qui ne signifie pas seulement "arriver», "se produire", "se réaliser ", "s'accomplir ", mais également " échoir : alicui », à quelqu'un : (Ibid., p. 44).

12 Ainsi l'événement surgit sans qu'il soit toujours visé par la conscience. En cela, Romano se distingue de l'approche husserlienne, au sens où le phénomène de l'événement ne trouve pas sa source dans l'intentionnalité de la conscience comme le pense Husserl, mais précisément excède la conscience. L'événement selon Romano (1998) : «(..) reconfigure les possibles qui le précèdent et signifie (...) l'avènement d'un monde nouveau » (Ibid., p. 51) et » tout événement véritable (...) bouleverse le monde en le reconfigurant » (Ibid, p. 55). L'événement porte en lui la possibilité de reconfigurer les réseaux de sens possibles; en cela, il bouleverse ce qui constitue mon horizon de sens, pour ouvrir d'autres sens possibles, sans nécessairement que ces derniers soient considérés, examinés par le sujet auquel advient cet événement. Dit autrement, le sujet humain peut vivre une expérience sensible comprise comme phénomène «événement» sans pour autant être en mesure de prendre en compte ce dernier. Certains sujets humains se saisiront de l'événement comme d'une occasion opportune pour reconsidérer l'ensemble de leur vie, y compris dans leur rapport aux autres et au monde; d'autres, seront possiblement submergés par lui, voire, engloutis. Le pouvoir d'agir des sujets humains est mis en jeu dans une telle expérience vécue. Dès lors, l'accompagnement éducatif et/ou thérapeutique se présente alors comme une aide afin que le sujet advenant puisse se ressaisir de ce qui lui arrive. Cette idée résonne avec cette citation de Romano (1998) : « tout événement ultérieur [à la naissance] est (...) la possibilité donnée à l'advenant de renaître » (Ibid., p. 111).

13 Il s'avère impossible de repérer a priori et en extériorité, quel phénomène en tant qu'expérience vécue relève ou non d'un événement, précisément parce que la sensibilité à cette expérience relève du sujet humain dans la singularité de son histoire et de son être. En revanche, a posteriori il sera possible de qualifier une expérience sensible de phénomène événemential. En effet, l'expérience comme événement qui advient et duquel surgit un déploiement de potentialités se repère grâce aux changements induits en considération du parcours suivi jusque-là et des récits faits par le sujet. Cette considération conduit à repérer un distinguo entre l'événement qui se vit dans l'expérience sensible comme surgissement phénoménal et les effets de cet événement, ses conséquences aussi bien en termes d'existence qu'en termes d'apprentissages liés à cette ex-per-ience. Pour le signifier autrement, l'événement se vit mais s'appréhende de façon médiate par les retours sur cette expérience via différents langages. Dans le cas de l'expérience douloureuse ou bien encore d'un traumatisme, faire retour sur l'événement difficile ou traumatisant nécessite parfois d'autres voies que la seule parole ou le langage verbal, la musique, les arts visuels, le modelage..., qui permettent de construire du sens. Nous retrouvons ici l'apport du concept d'automédialité développé par Delory-Momberger \& Bourguignon (2020) dans le cadre de la recherche biographique, lorsque le vécu expérientiel sensible d'un événement est tellement fort, qu'en parler avec des mots est parfois impossible indicible - en tout cas, réducteur. Tâcher de l'appréhender nécessite alors de recourir, voire de combiner un médium à un autre et de prendre le temps d'un apaisement des émotions. 


\section{L'événement sous le prisme d'une double temporalité à la faveur d'une raison au grand cœur}

Lesourd (2009) a travaillé avec finesse sur les notions de transition et de tournants de vie. En 2019, il rappelle la bipartition temporelle de l'événement (Denzin, 1989) pour désigner celui, premier, de l'expérience vécue avec une plus ou moins grande intensité émotionnelle ; puis celui, de la réception sociale et de la mise en sens. Il précise que :

C'est ce second temps qui permet de fonder rétrospectivement l'événement comme tel, de contresigner son existence et de l'élaborer à travers des interactions. En l'absence de réception sociale par un collectif ou un autre significatif, par exemple en cas de déni (il ne s'est rien passé) ou de disqualification (ce n'est pas digne d'intérêt), l'événement ne peut advenir pour le sujet : son premier temps ne faisant pas l'objet d'une reprise, il tombe hors histoire : (Lesourd, Ibid, p. 81).

L'événement suppose ainsi l'articulation d'un collectif au "soubassement sensible » de cet événement au niveau du sujet sous peine de tomber « hors histoire ». Mais de quelle histoire s'agit-il ? Ou n'y-a-t-il pas plutôt à considérer des niveaux d'histoire ? En outre, la non reconnaissance au niveau social efface-t-elle pour autant l'événement existant, vécu par le sujet? N'est-ce pas davantage les niveaux de résonnances symboliques et de construction de sens qui diffèrent sans pour autant annuler l'événement qui a surgi au niveau du sujet? N'y-aurait-il pas lieu de considérer l'approche développée par LanyBaile \& Mallet (2006), qui en écho à la pensée de la complexité de Morin, considère l'événement à différentes échelles : depuis celle sociétale et historico-politique, jusqu'à celle inter psychique et intrapsychique, non sans établir de liens entre elles?

\section{Traits caractéristiques de l'événement}

Romano (1998), ayant introduit une distinction entre l'explication des faits en rapport avec l'événementiel et leur compréhension au sens événemential, pose le caractère primordial des événements. Précisément, ce sont les événements qui transforment les sujets auxquels ils surviennent en advenants. En cela, ils participent fondamentalement à leur processus de subjectivation. Certes l'écho social, voire politico-historique, qui leur sera donné, vont contribuer à donner de l'ampleur en termes de socialisation notamment, mais son absence n'empêchera pas son existence ni ses résonnances sur l'advenant. Comme le rappelle Marcelo (2016), l'événement, selon Romano, possède quatre traits :

1) son assignation univoque (il s'adresse toujours à moi-même, m'interpelle et me bouleverse), ce par quoi il me confère mon ipséité ; 2) il est instaurateur de monde, en ce que, après son avènement et ma compréhension rétrospective de son sens plus rien n'est jamais le même pour moi ;3) il est constitutivement an-archique car il s'excepte de toute archéologie causale (non pas en tant que fait intramondain, ce qu'il est aussi sous un autre mode) dans la mesure où, d'un point de vue événemential, il est sa propre source ; 4) il n'est pas strictement datable dans le temps (encore une fois en tant qu'événement, malgré sa datation comme fait) mais, par son arrivée même, il ouvre le temps à partir duquel l'aventure fera sens: (Marcelo, Ibid., p. 172).

17 Autrement dit, l'événement possède un double rapport au temps; d'un côté, comme fait objectivable, il est datable et peut rentrer dans une explication selon une logique de causalité ; de l'autre, parce qu'il est considéré sous l'angle de son événementialité il n'est pas datable précisément et se trouve pris dans une trame temporelle dialectique 
entre son surgissement qui crée potentiellement une ouverture aussi bien sur le présent et l'à venir de l'advenant que rétrospectivement sur son passé. Cela a pour conséquence, une reconsidération du sujet humain, moins vu comme absolu préalable et premier à l'instar du cogito cartésien, que comme fondamentalement en construction et en relation avec les événements qu'il vit. Romano (2010) précise encore :

La phénoménalité de l'événement est indissociable à chaque étape de l'analyse, de la manière même dont l'advenant se l'approprie, l'intègre à une expérience (ou échoue à le faire), c'est-à-dire advient à lui-même à travers son épreuve : les faces «objectives » et « subjectives » de la description demeurent structurellement liées : (Ibid., p. 35).

18 À l'instar de Husserl, Romano (2010) considère la double dimension objective et subjective d'un phénomène et signifie que l'expérience possède un logos immanent, anté-prédicatif, que la phénoménologie a précisément pour but de mettre au jour sans réduire l'expérience à ses aspects objectivés. En outre l'ipséité de l'advenant se comprend comme sa capacité à reconfigurer son soi en lien avec l'événement dans son événementialité c'est-à-dire dans la reconstruction de sens auquel celui-ci peut conduite étant donné que par son irruption, il ouvre le temps et conduit à réinterroger son rapport à soi, au monde, aux autres. L'herméneutique événementiale de Romano renverse le rapport entre l'être-au-monde et les événements, développant ainsi une nouvelle compréhension du sujet humain dont le processus de subjectivation passe par les événements et contribue à faire place à l'expérience dans ce qu'elle a d'infrarationnel. Comme le formule Romano (2010) :

C'est à une raison élargie que Husserl entend nous reconduire en nous ramenant " aux choses mêmes ». C'est raison au grand cœur, qui situe le cœur de la raison dans les relations de la pensée au sensible, qui constitue l'objet de ses recherches: (Ibid., p. 15).

19 Tâchons à notre tour de considérer cette « raison au grand cœur » comme l'appelle Romano, cette raison élargie, qui inclut la sensibilité. Marin (2019) dans son ouvrage intitulé Rupture(s) évoque les blessures de la vie que nous traversons tous, pouvant entraîner des ruptures dans le cours de la vie, qu'il s'agisse des ruptures voulues ou de celles que l'on subit. Et, «[s'] il faut parfois rompre pour "se sauver », c'est-à-dire à la fois fuir et sauver sa peau » (Ibid., p. 14), Marin précise qu'elle résiste à une approche optimiste, voire positiviste, qui attribue rétrospectivement toujours une valeur positive à un " échec ». Car, ce changement est toujours susceptible de se retrouver à nouveau «dans des chemins de traverse. [Tant] Il est possible que je n'aie finalement rien appris » (2019, p. 21).

En définitive, Romano $(1998,2010)$ dans le sillage de Husserl considère la possibilité d'une saisie pré-linguistique de la chose même, dans une saisie intuitive à laquelle contribue le champ des perceptions. Dans ce cadre, "l'expérience du monde est nécessairement celle d'un sujet en sorte qu'il n'y a pas plus de sujet sans monde que de monde sans sujet » (Cabestan, 2016, p. 13). Ainsi, l'expérience ne saurait être seulement subjective car elle renvoie également à autre chose que les seuls éprouvés et vécus de conscience du sujet (Romano, 2010, p. 804). Dit autrement, le sujet n'est pas la seule source de sens. Il s'opère alors non seulement une réhabilitation du monde sensible dans le domaine de la raison, que Romano appelle "une raison au grand cœur» (Romano, 2010, p.14), mais également une prise en compte du monde de la vie, la Lebenswelt de Husserl (2008). Pour autant, il est des événements qui traumatisent, et qui 
laissent parfois sans voix, ceux qui les vivent ou les éprouvent comme tel. Que dire alors de leur dimension et de leur herméneutique événementiale?

\section{Evénements traumatiques}

21 Les événements traumatiques font écho à ces épreuves, pour lesquelles le sujet humain ne se remet pas ou difficilement. Considérons dès lors ce que nous pourrions en comprendre, notamment au plan d'une recherche biographique. Les traumatismes en tant qu'événements sont certes uniques, irréversibles et singuliers, mais leurs expériences résonnent au plus profond des êtres, quand bien même leurs sens ne sont pas - encore - construits par eux ou relayés par les autres, le groupe, la société. Nous pourrions ainsi dire, que l'événement traumatique peut constituer d'un côté, un fait datable et situé dans le temps et l'espace, lorsqu'il est considéré sans prise en compte de ce qu'il produit au niveau de l'intériorité du sujet auquel il advient; et qu'il est, de l'autre, un événement-ex-pér-ience dès lors qu'une attention est portée à ce que vit, éprouve et apprend le sujet humain à qui advient cet événement. L'événement-ex-périence comporte une durée, qu'il est difficile de dater avec précision notamment parce qu'il touche à l'infra-conscient du sujet advenant. Pour autant, il s'inscrit dans l'histoire du sujet, qui ne se départit pas du rapport au monde et aux autres. Cet ancrage théorique fait également sens avec les travaux en recherche biographique de DeloryMomberger (2009) concernant la condition biographique. En effet, le processus de biographisation compris comme processus par lequel les sujets construisent la figure de leur existence et la reconfigurent en fonction des événements, ne saurait faire abstraction des modes historiques et culturels d'existence individuelle et collective, propres aux sociétés contemporaines. Avec l'appui de la pensée de Romano, nous pourrions réfléchir au traumatisme comme ex-pér-ience limite, comme ex-pér-ience de «l'inassimilable».

Les recherches réalisées sur les terrains conduisent parfois aux limites de ce qui peut se dire lorsque le sujet humain se trouve en situation de vulnérabilité telle l'exclusion, le handicap vécu comme ostracisation, voire, le rejet de la part des autres, ou bien la maladie, de celles qui portent atteintes à la vie elle-même, ou bien encore lorsque l'intégrité de la personne et sa souffrance sont tels, qu'il devient difficile de vivre et d'en parler. Le traumatisme nous semble emblématique de ces situations difficiles à vivre, précisément parce que les récits entendus lors des réalisations d'entretiens avec les personnes en situation de handicap ${ }^{1}$, les personnes malades chroniques ${ }^{2}$ et les proches ${ }^{3}$ (parents ou conjoint.es) de personnes dites en «état de conscience altérée " (pauci-relationnel ou état végétatif chronique) renvoient toutes à des situations de recherche où l'émotion affleure d'emblée.

23 Avec la radicalité et la force qui caractérisent le traumatisme d'une façon ou d'une autre, le sujet humain vit cette expérience comme un événement à caractère disruptif, qui le marque au point parfois non seulement de transformer sa vie, son rapport aux autres et au monde qui l'entoure, mais également de transformer ses proches. Un seul événement et subitement ne plus être tout-à-fait le même, plus tout-à-fait soi, qu'il s'agisse de la personne qui a subi le traumatisme en direct ou qu'il s'agisse d'un dommage collatéral pour les proches, c'est en effet l'ipséité qui est touchée, ébranlée et plus ou moins anéantie. Le traumatisme serait-il alors paradoxalement l'événement par excellence au sens «événemential» dans la phénoménologie de Romano $(1998,2010)$ 
tout en étant le moins propice à sa description, dans la mesure où souvent, il sidère les personnes au point de plus pouvoir en parler? Que peut apporter une approche phénoménologique du traumatisme? Et si, comme l'écrit Lorelle (2016): «loin de [seulement] signer l'échec de la mondanéité comme de l'ipséité en marquant la limite de toute description phénoménologique, [le traumatisme] en révèle bien plutôt une dimension oubliée, plus sensible que toute sensibilité, au-delà de tout pouvoir et de toute identité ?» (2016, p. 48).

Sans être en capacité de faire une synthèse théorique des travaux existant en psychanalyse et en psychiatrie sur cette notion, les premières recherches bibliographiques indiquent que les publications sur le traumatisme se déclinent sous de nombreuses formes notamment dans le cadre de la santé mentale. Il est moins question de traumatisme que de trauma en lien avec telle ou telle rubrique de santé ou de tel objet de traumatisme. Néanmoins, pour tenter d'approcher ce qui caractérise le traumatisme indépendamment de telle ou telle dimension particulière et singulière, nous allons recourir à quelques références incontournables de ce qui est nommé traumatisme à dimension collective. Ferenczy (1927/2006) appréhende le phénomène du traumatisme comme un "choc", où se joue un "anéantissement du sentiment de soi » (Ibid., p. 33) portant atteinte à la capacité de résister, d'agir, voire de penser.

Il se peut aussi que les organes qui assurent la préservation de soi abandonnent ou du moins réduisent leurs fonctions à l'extrême. (Le mot Erschütterung [commotion psychique] vient de Schütt [débris]; il englobe l'écroulement, la perte de sa forme propre et l'acceptation facile et sans résistance d'une forme octroyée, 'à la manière d'un sac de farine' : (2006, Ibid., p. 33).

Il précise également d'autres caractéristiques du traumatisme :

$[. ..] 1^{\circ}$ le cours de la paralysie sensorielle sera et restera durablement interrompue ; $2^{\circ}$ durant celle-ci on acceptera sans résistance toute impression mécanique et psychique $; 3^{\circ}$ aucune trace mnésique ne subsistera de ces impressions même dans l'inconscient, de sorte que les origines de la commotion sont inaccessibles par la mémoire. Si toutefois on veut les atteindre (ce qui semble à peu près impossible), alors il faut répéter le traumatisme lui-même, et dans des conditions plus favorables, l'amener, pour la première fois, à la perception et à la décharge motrice : (2006, Ibid., p. 40).

Le refus de «s'adapter à la situation de déplaisir évoquée par Ferenczy, qui « exige une soupape " peut prendre forme d'" autodestruction qui, en tant que facteur délivrant de l'angoisse, sera préférée à la souffrance muette » (Ibid., p. 36). Rappelons également le propos de Chiantaretto (2009), lorsqu'il rappelle combien : «Parler sa vie à quelqu'un lorsque le semblable a été attaqué en soi : telle est la tâche du témoin survivant, l'enjeu étant radicalement de rendre sa vie vivable » (Ibid., p. 111).

D'autres auteurs précisent l'importance de l'anxiété lorsque la maladie grave, par exemple, s'invite durant l'enfance (Van Broek \& Rillaer, 2012) et l'adolescence (Dauchy \& Razavi, 2010). Ils insistent alors afin que celle-ci soit prise en compte au même titre que la douleur, afin que les jeunes gardent confiance non seulement en eux-mêmes, mais également envers les adultes, de façon à pouvoir développer leur personnalité de façon harmonieuse. 


\title{
3. Face aux événements éprouvés : quelle posture pour le chercheur?
}

28

\begin{abstract}
bien plutôt aux côtés de la personne qui éprouve avec souffrance, un événement. Qu'en est-il en effet de la recherche et du chercheur impliqué dans le dialogue «je » / « tu», qui engage comme nous l'avons vu, des dimensions d'intériorité et d'extériorité ? Car, il est des problématiques de recherche qui conduisent le chercheur aux bords du trauma vécu par les personnes auxquelles il s'adresse et qui ne manquent pas de l'atteindre en retour. Quelle(s) précaution(s), le chercheur adopte-t-il alors, sachant qu'il n'est pas thérapeute et que sa propre sensibilité interfère avec celle de la personne avec laquelle il interagit en recherche?
\end{abstract}

La question se pose de savoir comment, en tant que chercheur se positionner face ou

En recherche biographique (Delory-Momberger, 2014), étant donné qu'il s'agit de reconnaître et de prendre en compte le sujet humain, ses expériences notamment grâce à ses récits oraux ou bien écrits, appréhendés comme traces éprouvées de soi, traces d'un «savoir que » pouvant ou ayant déjà permis une formation de soi (Bildung), les expériences vécues comme traumatiques nécessitent une réflexion éthique sur la posture du chercheur.

\section{Solliciter un récit en adoptant une posture éthique}

30 En premier lieu, solliciter la reconfiguration d'un événement vécu comme traumatique (indépendamment de la nature par une personne, à travers l'élaboration d'un récit nécessite une posture éthique chez le chercheur d'autant que ce dernier considère comme fondamentale, la valeur cardinale du respect de la personne humaine, précisément celle sans laquelle aucune relation humaine digne n'est possible. Cette posture éthique se retrouve également revendiquée dans le monde du soin. Laub, par exemple, invite à considérer combien :

\begin{abstract}
(...) le fait que le défaut massif de relais des besoins par l'environnement, comme lors d'un génocide, questionne l'existence même de l'empathie, de la communication humaine et jusqu'à notre propre humanité quand les miroirs cessent d'exister. Une telle expérience de vie représentera, pour le survivant du trauma, l'échec d'une réponse empathique de la part de quiconque, agent ou fonction. Parce que, précisément, la représentation des interactions de satisfaction des besoins est à la base des liens entre l'existence individuelle et le lien social, l'attaque pour un individu de la représentation d'un environnement qui relaie les besoins déconstruira le lien entre soi et l'autre. La destruction, chez la victime, de la matrice où se représentent les relations interpersonnelles entraîne une vulnérabilité et une solitude dans la représentation de son monde interne, qui est la condition sine qua non d'un trauma perpétré par l'homme » (Laub, 2015., p. 29).
\end{abstract}

Dans le cadre de nos propres travaux en lien avec différentes enquêtes de terrain, nous avons repéré la nécessité d'une réflexion éthique tout au long de la recherche ellemême; distincte en cela de la seule application d'un code déontologique de bonne pratique en recherche qualitative (Janner Raimondi, $2015 ; 2017$; 2018 ) pour entrer en relation avec la personne directement concernée par l'enquête de terrain. Cette nécessité éthique conduit à tâcher de se montrer tout-à-la-fois humble, à l'écoute, empathique et prudent pour limiter le risque de blesser la personne tout au long des échanges, y compris après lorsqu'il s'agit d'écrire la recherche. Il s'agit en réalité d'une 
d'éthique de la délicatesse, particulièrement attentive à la personne avec laquelle le chercheur entre en dialogue dans le cadre d'une recherche comprise avant tout comme rencontre humaine dans laquelle le chercheur porte toute la responsabilité. Après réflexion, il est apparu: d'une part, que l'empathie, entendue comme processus se déroulant in situ au cœur des échanges lors de la rencontre avec l'autre, ne saurait être le fait d'un seul des interlocuteurs. C'est tout l'enjeu d'une empathie appréhendée dans la réciprocité des échanges. Cette empathie se noue au fil de la conversation, aussi bien du côté de la personne, auprès de laquelle l'entretien se réalise, que du côté du chercheur, qui invite à raconter. D'autre part, la spécificité de l'empathie consiste à chercher à comprendre l'autre en essayant d'adopter son point de vue, c'est-à-dire de là où il est, et non en gardant son point de vue propre de chercheur en extériorité. Ceci a conduit, comme l'ont précisé Berthoz (2004) à élaborer un changement de cadre de référence de façon à opérer une rotation mentale propre à rendre possible l'adoption d'un point de vue différent du sien initial, sans jamais pour autant confondre son point de vue avec celui de cette personne. Enfin, nous avons repéré que ce processus empathique, outre les dimensions cognitives qu'il déploie permettant de construire du sens sur ce qui est dit, s'appuie également sur des éléments du monde sensible, notamment en prenant en compte, les signes du corps, le ton de la voix, les postures physiques et corporelles comme autant d'éléments de langage verbaux et non verbaux permettant d'être continument attentif à l'autre. Faire preuve d'empathie, comme l'ont souligné de nombreux auteurs (notamment : Sironi, 2004 ; Tisseron, 2010 ; 2014 ; Zanna, 2015), renvoie ainsi à la nécessité pour le sujet humain d'établir un lien de confiance avec les autres et le monde, non seulement au regard de sa néoténie, mais également en rapport avec le fait de pouvoir vivre ensemble et de «se sentir chez soi dans le monde » (Amery, 1995).

Pour autant, le chercheur en sciences de l'éducation et de la formation (SEF) ne saurait se confondre avec le thérapeute, précisément parce que ni le but, ni les méthodes ne sont les mêmes. Si la posture éthique visée paraît identique, l'enjeu de la recherche en SEF n'est pas d'intervenir au nom d'un traitement thérapeutique ou d'un soin, ce qui entraîne des compétences et des connaissances spécifiques. Si le verbe "guérir " signifie non pas le retour à un état antérieur à la maladie ou au trouble, mais bien plutôt une forme de vie viable pour tous, le chercheur en SEF ne saurait volontairement se donner comme objectif de contribuer à "guérir » la personne avec laquelle il va s'entretenir : d'une part, parce que lui-même a à apprendre de la personne rencontrée ce qui est moins le cas du thérapeute qui développe un diagnostic/pronostic sur la base de connaissances objectives du trouble considéré ; d'autre part, parce que son objectif fondamental vise à produire et diffuser un savoir sur une question donnée, en reconnaissant la valeur des savoirs issus de l'expérience de cette personne. Ce dernier renvoie à la double herméneutique de Paillé (2020) :

(...) l'acteur incarne une première herméneutique (il a sa propre interprétation de sa vie intime et sociale) que le chercheur replace dans une deuxième herméneutique (faite de ses référents théoriques et expérientiels ainsi que de son interprétation des conduites étudiées. Pour le chercheur comme pour l'acteur, la rencontre de l'autre est un événement dont le caractère herméneutique rappelle qu'il participe de la complexité des processus de la compréhension et de l'interprétation. (...) La rencontre de l'autre est, dans ses prémisses comme ses conclusions, aussi une rencontre de soi. Ce que nous sommes s'active, se révèle et profite dans la rencontre ; ce que nous devenons s'enrichit du gain de connaissance de soi qu'éveille en écho la vie de l'autre : (Paillé, Ibid., p. 224). 
Pour autant, comme le soulignent encore de Villers \& Niewiadomski (2002), solliciter le récit de soi à travers l'autobiographie peut avoir des effets "thérapeutiques" involontaires, dans la mesure même où ce n'est pas le chercheur qui les vise en tant que telles, mais c'est la pratique du récit de son expérience et la formulation des savoirs qui lui sont liés qui combine trois dimensions spécifiques: épistémique, formative et thérapeutique. En effet, pour de Villers (2011):

[Car] faire son récit de vie libère le sujet qui l'énonce pour un projet dont la détermination est rendue possible par la construction même de son histoire en récit. Ainsi, produit du discours narratif, le sujet peut s'inventer un avenir sur la base de ce qu'il a pu élaborer de son histoire. C'est ainsi que nous croyons pouvoir repérer l'effet de relance que peut produire l'élaboration d'un récit, effet de relance pour le projet de vie, pour le projet professionnel et, finalement, pour le projet de soi-même : (2011, § 17).

Ainsi, la recherche biographique, parce qu'elle sollicite une rencontre initiant un dialogue au cœur d'une relation éthique de respect donne potentiellement forme à un "prendre soin de soi », dans le sillage de la déclinaison du prendre soin propres aux éthiques du Care, en référence à Tronto (2009).

\section{Une posture de recherche qui vise la délicatesse en se réfèrant au senti/sentant}

Si l'éthique exige la valeur du respect, celle-ci demande à être déclinée et traduite dans les faits. Nous avons parlé de l'empathie, mais nous aurions tout autant pu convoquer les notions de délicatesse et de tact.

Vincent-Buffault (2019) définit la délicatesse comme ce qui tient :

(...) du ténu et du délié : elle suggère à la fois la finesse, la douceur et le tact [...]. Elle est une manière de sentir et en appelle au doigté, au flair, au tact qui impliquent de ne pas trop toucher, conviant au dessaisissement au cœur de l'approche Elle permet d'accueillir la vulnérabilité sans s'appesantir : (2019, p. 182).

En écho à l'esprit de finesse de Pascal, la délicatesse mêle sensibilité et pensée. Elle laisse percevoir une pensée qui perçoit, comprend et se laisse toucher dans les infimes nuances de l'affleurement sans peser. Les savoir-être, savoir-faire et savoir-dire propres à la délicatesse se traduisent dans des attitudes d'écoute perceptible et attentionnée, capable de se mettre au diapason, sans toujours très bien comprendre d'emblée. Ces savoirs rendent perceptibles un regard attentif sans se montrer appuyé et rendent capables de proférer une parole ajustée à la personne, aux circonstances et à l'événement narrés comme aux éprouvés qui émotionnellement s'expriment alors. Comme le précise Hesbeen (2018), la délicatesse fait partie de ces mots qu'il qualifie de " fragiles », non en raison de leur " faiblesse » mais de leur légèreté ». Ainsi, l'humilité, la générosité et la délicatesse ont pour lui des valeurs du prendre soin et renvoient au « juste sens de la finesse ». La délicatesse se situe entre le « peu » et le « assez »: « trop peu n'en est pas ; trop n'en est plus, c'est un juste sens de la finesse » (2018, p. 34).

La délicatesse prend forme de tact articule les trois désinences du mot: le sens du toucher ou la modalité de la sensibilité; la faculté de juger intuitivement ou bien encore l'appréciation fine de ce qu'il convient de dire ou de faire en matière de goûts, de convenances ou d'usages. 
39 La réflexion de Merleau-Ponty (1945) permet de comprendre combien la perception est multimodale et ne saurait se cibler isolément sur un sens. Le toucher s'entend alors sur un versant passif "être touché» et sur versant "actif toucher», qui par-delà les modalités physique et sensorielle, s'appréhende également au plan symbolique. Ainsi, nous pouvons, par résonnance et interprétation, entrer en contact symbolique avec autrui. Clerget (2006) précise également combien le toucher est «polyphonique à souhait ", s'ouvrant à " une topologie où s'articulent le dehors et le dedans » (2006, p. 19). Dès lors, nous comprenons mieux l'idée de Levinas (1974) selon laquelle « le contact n'est pas ouverture sur l'être, mais exposition à l'être» (1974, p. 128). Le toucher par le contact qu'il engage, y compris au plan symbolique, se retrouve dans toute expérience sensible par le fait même que le corps de chair se vit dans l'entrecroisement du "être touché » et du toucher, du dedans et du dehors, du sensible et du sentant. Comme le formule encore Clerget: "le toucher a cette particularité que je ne puis toucher sans être touché, ce qui lui confère sa portée d'emblée interprétante" (2006, 24). C'est pourquoi, l'auteur attribue au toucher les deux versants actif et passif de notre réceptivité symbolique dès lors que s'établit une relation entre des personnes.

40 Les travaux de Berger (2006) et Austry \& Berger (2014) permettent de comprendre le toucher en tant que canal empathique spécifique particulièrement aidant dans le cadre du soin et de l'accompagnement, mais également comme lieu de résonnance intime et comme source de connaissance. "Le toucher est dans sa fonction première, un sens de la relation et de la relation au monde » $(2014,231)$, par lequel non seulement je me confronte mais aussi par lequel je rencontre, découvre, apprivoise cette réalité. Reprenant la double dimension selon laquelle pour toucher il faut être touché, que les auteurs nomment doublitude $(2014,232)$ pour signifier la co-existence mutuelle, voire l'entrelacement des deux (Merleau-Ponty), à l'exemple de la main touchante et de la main touchée déjà présent chez Husserl (1929/2008). Ces réflexions conduisent à penser que l'empathie, en tant que processus de compréhension d'autrui, qui passe par les modalités multi sensorielles, imaginatives et cognitives de la conscience sous forme analogique, peut s'appréhender comme toucher symbolique.

41 La notion d'événement appréhendée du point de vue événemential permet ainsi de s'ouvrir à l'expérience sensible vécue permettant d'interroger et de réinterroger le sens de ce qui est advenu et de ce qui continue d'advenir aussi bien pour la personne considérée, que pour le chercheur qui rencontre et dialogue avec celle-ci. En cela, il ne s'agit pas seulement de faire usage d'une raison instumentale, qui rationnaliserait ce qui s'est passé pour la personne concernée. Il s'agit bien plus de considérer les enjeux éthiques et épistémologiques, voire, formatifs, d'une prise en compte en recherche de ce que Romano a appelé la raison au grand cœur dans laquelle interfère l'historicité du processus de subjectivation. Elle laisse également voir la personne humaine sous l'angle de son devenir, et non comme un noyau identitaire replié sur lui-même. Le chercheur, qui s'efforce d'apprendre de l'autre convoque, outre une conception du savoir ouverte à la reconnaissance de ce que les personnes tirent de leurs expériences, une posture éthique, emprunte de délicatesse et s'efforçant avec tact et subtilité de se laisser toucher et de comprendre dans un "juste sens de la finesse », ni trop pesant, ni pas assez. Inévitablement, les travaux issus de telles recherches ne sauraient manquer ni de garder trace, ni de chercher à rendre compte des traces de sensibilité, qui ré-ouvre ainsi le partage initial du sensible. 


\section{Présentation du numéro}

42 Les articles qui suivent s'inscrivent dans une réflexion inhérente aux entrelacs des expériences vécues, de leurs éprouvés et de ce que les personnes en disent aussi bien en leur nom propre qu'au niveau des chercheurs qui s'efforcent de rendre visibles ses dimensions dans leurs écrits scientifiques.

En exergue, Christophe Blanchard donne à entendre le témoignage d'un projet qui vise à permettre aux personnes de se réapproprier les évènements de leur propre histoire pour donner à voir qui elles sont, par-delà le curriculum vitae habituel occultant les aspects pouvant relever de l'intime, afin d'accéder à un emploi, notamment en lien avec le travail réalisé par la start-up sociale Whire, en prise avec le parcours biographique des personnes.

Une première partie développe une réflexion à la fois théorique et étayée sur des recherches empiriques concernant les expériences vécues dans le sensible des corps-de-chair aux événements et leur historicité.

Martine Janner Raimondi s'efforce de considérer les fondements théoriques propres à rendre compte de la possibilité d'une intercompréhension autant au niveau des chercheurs, que des personnes concernées par une étude et des lecteurs. L'approche phénoménologique et herméneutique de l'expérience vécue propose ainsi un cadre pour la recherche biographique en éducation-formation, qui invite les chercheurs recourant aux récits biographiques à appréhender les enjeux souvent infra perceptibles du corps de chair pris dans un rapport aux autres et au monde commun ouvrant ainsi la voie d'une connaissance hybride où se tissent rationalité scientifique, subjectivité et intersubjectivité.

Frédéric Moussion, quant à lui, s'appuie sur le récit comme la mise en relation d'un ensemble d'énoncés et d'événements ayant trait aux expériences du locuteur. Les phénomènes d'hyper-correction concernant les activités d'auto-contrôle eu égard aux «bons » usages d'une langue seconde, peuvent être considérés comme des événements en rapport avec le sentiment d'insécurité linguistique. Les phénomènes d'hypocorrection, considérés a priori comme des non-événements, s'avèrent in fine comme des événements pouvant renvoyer lorsqu'ils sont revendiqués et assumés à des actes révélateurs d'un pouvoir d'émancipation du sujet.

Izabel Galvao développe, quant à elle, un retour réflexif sur une démarche de recherche-collaborative menée au sein d'un centre d'hébergement et stabilisation (CHS), dont l'objectif visait à soutenir l'équipe de salariés dans la préparation du déménagement de ce centre accueillant des personnes sans-abris, grâce à la coélaboration d'un récit collectif faisant place aux acteurs. Restituer des éléments de ce récit collectif a permis de repérer combien par-delà l'événement inexorable du déménagement annoncé, la construction d'un lien entre les acteurs via l'élaboration d'une mémoire collective a permis de constituer un levier propre à développer un pouvoir d'agir aussi bien des personnes en situation de précarité que de professionnels en prise avec des conditions de travail et un secteur fragilisés.

Dans une deuxième partie, il s'agit de présenter des événements in situ de l'espace sensible mis en récit.

49 Marie-Hélène Verneris-Pétigny précise combien l'expérience du récit issu de situations de l'extrême ne saurait être vécues, ni pensées dans les termes d'un récit ordinaire. Sur 
la base de repères inscrits en psychanalyse et en psychologie, l'auteure étudie comment la recherche biographique en éducation-formation appréhende la notion d'événement traumatique, et comment cette dernière permet de comprendre la façon dont les événements marquants transforment le cours de la vie d'une personne lorsque s'entrecroisent des bifurcations et des expériences venant reconfigurer ses traits identitaires. Plus précisément, c'est à travers les récits de deux personnes incarcérées, que l'auteure montre par-delà leurs caractéristiques communes, toute l'importance de dire et du dit, compris comme acte de survivance, en écho au besoin essentiel de partager une histoire pour retrouver une dignité.

Ensuite, Carole Baeza, dans le cadre d'une recherche interventionnelle en santé, menée conjointement entre patients, hématologues et chercheurs en sciences de l'éducation et de la formation, présente l'itinéraire d'auto-soin d'un patient hémophile, appelé Paul. $\mathrm{Au}$ cours d'un entretien biographique, il décrit son processus actif de prophylaxie et, notamment, comment il prend la décision de se soigner suite à une perception interne pour laquelle il pressent que quelque chose ne va pas dans son corps. Les liens entre des signes infra-cliniques, avant-coureurs d'une crise, et ce qui fera ultérieurement événement, sont rendus perceptibles à travers des mots, mais surtout des métaphores, qui révèlent ses apprentissages issus de ses expériences du vivre au quotidien avec la maladie. Ainsi, les éprouvés sensibles du corps, rendus dicibles, grâce à divers registres de langue allant de l'expression des perceptions, sensations, notamment via des images visuelles et des métaphores donnent corps aux apprentissages et révèlent à quel point repérer ce qui fait évènement dans son corps propre peut constituer un atout majeur en éducation thérapeutique du patient.

51 Puis, Mike Gadras examine à l'aune du concept d'évènement les dynamiques de sens et d'action qui entourent les conditions de vie d'un collectif composés de 163 exilés, dits "sans-papiers", installés depuis dix dans une usine désaffectée. Cette enquête ethnographique réalisée durant 5 ans donne à entendre comment construit le sens d'un événement et comment se développe l'attention à l'autre, notamment au regard d'un questionnement social. Prenant appui sur l'approche théorique de Romano (2018), l'analyse des évènements intra-mondains, qui traversent et structurent les expériences individuelles et collectives de ces exilés, débouche sur une «herméneutique événementiale ", qui vise à rendre compte non seulement des formes de recomposition sociale dans les marges mais également des processus de subjectivation à l'œuvre au cœur des expériences de migration irrégulière, en contexte de région parisienne.

52 Enfin, Valérie Melin précise combien les problématiques de décrochage/raccrochage scolaire s'inscrivent dans une historicité à la fois individuelle et sociale qui convoque les représentations des personnes aussi bien au niveau de ce qu'elles sont, que d'où elles viennent et ce qu'elles souhaitent. Le passé scolaire et le vécu de la rupture avec l'école, souvent difficiles à élaborer du fait de la souffrance psychique qui leur est associée, pèse autant sur le travail de (re)construction du rapport au savoir que sur l'expérience du raccrochage, lui-même, tourné prioritairement vers un projet. La démarche de narration de soi, comme travail biographique, permet à la personne, dans les conditions de son inscription sociale, d'intégrer, de structurer, et d'interpréter les situations et les événements de son existence. Explorant les conditions à la fois cliniques et politiques d'un processus de subjectivation fondé sur une dynamique de distanciation réflexive à l'égard de l'expérience du décrochage, l'article envisage de 
dépasser la dimension traumatique de l'événement décrochage afin de rendre possible et d'ouvrir un à venir.

\section{Dans une troisième partie, il s'agit d'approcher et dire les événements du point de} vue des méthodes de recherche et des formations

Anne Dizerbo revient sur une expérience de recherche liée à la maladie chronique rare auto-immune dont le but consistait à mettre au jour et à rendre compte de l'expérience sensible des malades au niveau du vivre avec la maladie et ses traitements. La démarche initiale renvoyait à une méthodologie précise et éprouvée dans le cadre d'un autre contexte avec une pathologie différente. La perspective de l'article permet de réfléchir aux enjeux de recherche propre à une recherche biographique en éducation/ formation, qui s'appuie notamment, sur la subjectivité des personnes malades, à identifier et caractériser les ressources qu'elles déploient sans oublier les difficultés rencontrées dans le but d'améliorer leur prise en charge et d'élargir les propositions en termes d'éducation thérapeutique. Prenant appui sur la mise en récit des expériences par le biais d'une e-plate-forme collaborative envisagée au départ comme favorable au partage et à la co-construction de savoirs, il est apparu nécessaire de réexaminer les choix méthodologiques mis en œuvre pour prendre en compte les dimensions sensibles tant au niveau des enjeux de posture de recherche qu'au niveau des restitutions respectueuses des personnes et de leurs acquis d'expérience.

Julia Midelet et Valérie Viné Vallin précisent, quant à elles, combien parler, écouter et poser des questions pour recueillir le point de vue de la personne avec laquelle des entretiens compréhensifs sont prévus s'avère in situ, bien délicat, par-delà les ouvrages et recommandations méthodologiques, notamment lorsqu'il s'agit de s'entretenir avec des jeunes porteurs d'une déficience intellectuelle, éprouvant des difficultés à entrer en communication, à mobiliser leurs souvenirs ou encore à affirmer leurs idées et leurs choix propres. Peu sollicitées habituellement, ces personnes viennent bousculer les pratiques et démarches d'enquête par entretiens, obligeant le chercheur à trouver les moyens de s'ajuster en changeant d'outil d'enquête, de supports et de posture. Repenser la méthodologie adoptée jusque-là, afin de veiller à "prendre soin» de la relation à l'autre, pour prendre en compte la singularité de ces personnes, en imaginant comment favoriser leurs prises de parole sans risquer de blesser, tout en se montrant lucides sur les risques qui peuvent advenir durant les échanges - allant parfois jusqu'à infléchir leur parcours suite aux entretiens-; telles sont les éléments abordés dans cet article.

Puis, Marie-Hélène Verneris Petigny, qui intervenient régulièrement en milieu carcéral, auprès de personnes en situation de grande vulnérabilité, s'appuie sur son expérience, pour questionner les différentes postures du chercheur confronté à des situations sensibles. Que penser de sa place et de son rôle en tant que chercheur.e, face aux expériences transmises? Le terrain sensible du sensible invite le chercheur à reconsidérer son sens moral et sa crédibilité; il questionne fondamentalement sa responsabilité, sa légitimité, son objectivité, sans omettre de considérer sa capacité à entendre et voir la souffrance d'autrui sans pouvoir la réduire, ou bien encore, sa capacité à prendre parti face à l'institution qui les enferme. Aux conflits moraux ressentis par la chercheure face aux situations génératrices de malaises ou de troubles, s'ajoute une relation dissymétrique qui empêche la réciprocité entre égaux. $\mathrm{Si}$, comme le formule Rostaing (2010, p. 23) «l'on ne sort jamais indemne d'une recherche longue en prison" c'est moins le fait d'avoir été une femme parmi un groupe de quinze 
détenus, enfermés à double tour sans surveillance dans une salle de classe pendant plusieurs heures, que d'avoir été témoin de faits de violence inexplicables sur des personnes incarcérées qui interroge. Confrontée régulièrement à un univers de pratiques violentes, la chercheure s'interroge et, par-là-même, nous interpelle.

Enfin Séverine Colinet et Béatrice Mabilon-Bonfils approchent les questions de formation à la laïcité dans l'école de la République en prenant en compte les évènements traumatiques successifs qui font effraction dans le vivre-ensemble scolaire. La recherche dont il question dans cet article vise à présenter un dispositif d'ingénierie, appelé « ingénierie du bonheur », ancré sur le savoir-relation, qui mobilise l'expérience sensible des sujets pour soutenir la relation et guider l'action. Les entretiens biographiques réalisés auprès d'élèves et d'enseignants leur permettent de commencer à apprécier les effets du dispositif de formation. Pour ces auteures, accéder à l'expérience sensible de l'autre permet d'ouvrir l'espace des possibles, en entretenant un dialogue conçu comme espace "d'intéressement » (Akrich, Callon et Latour, 2006), dans lequel les dimensions biographiques et artistiques constituent des leviers majeurs.

\section{BIBLIOGRAPHIE}

Akrich, M., Callon, M., et Latour, B. (1991). L'art de l'intéressement, Dans Gestion de la recherche. Nouveaux problèmes, nouveaux outils. (pp. 27-52). Bruxelles : De Boeck.

Amery, J. (1995). Par-delà le crime et le châtiment. Trad. F. Wuilmart. Arles : Actes Sud.

Aristote (1991). Métaphysique, A, 1, 980 b. Trad. J. Tricot, tome 1. Paris: Vrin.

Auerhahn, N.C., Laub, D. \& Peskin, H. (1993). Psychotherapy with Holocaust survivors, Psychotherapy: Theory, Pratice, Training, 30 (3), 1993, 434-442.

Austry, D. \& Berger, E. (2014). Empathie, toucher et corps sensible : pour une philosophie pratique du contact. In : M. Botbol, N. Garret-Gloanec \& A. Besse (Dir.). L'empathie au carrefour des sciences et de la clinique. Cerisy : Ed. Doin, 229-246.

Berger, E. (2006). La somato-psychopédagogie. Où comment se former à l'intelligence du corps. Paris : Ed. Point d'appui.

Bernet, R. (2004). Conscience et existence : Perspectives phénoménologiques. Paris : PUF.

Berthoz, A. (2004). Physiologie du changement de point de vue. In: A. Berthoz \& G. Jorland (Dirs.). L'empathie. (pp251-275). Paris : Odile Jacob.

Botbol, M. Garrett-Gloanec, N. \& Besse, A. (Dirs, 2014). L'empathie au Carrefour des sciences et de la clinique. Cerisy : Ed. Doin.

Cabestan, P. (2016). L'événement et la raison. Autour de Claude Romano. Paris : Cercle herméneutique.

Chiataretto, J-F. (2009). Raconter pour vivre Survivre à un traumatisme, traumatisme de la survie. In : C. Delory-Momberger \& C. Niewiadomski (Dirs.). Vivre/Survivre Récits de résistance.

(pp. 111-117). Paris : Téraèdre.

Clerget, Joël. (2006). La main de l'autre. Le geste, le contact et la peau. Toulouse : Erès. 
Dauchy, S. \& Razavi, D. (2010). L'adolescent atteint de cancer et les siens : quelles détresses, quelles difficultés, quels souhaits d'aide? Paris: Springer Science \& Business Media.

Delory-Momberger, C. \& Bourguignon, J-C. (2019). Automédialité. In : C. Delory-Momberger (Dir.). Vocabulaires des histoires de vie et de la recherche biographique. (pp. 36-39). Toulouse : Erès.

Delory-Momberger, C. (2014). De la recherche biographique en éducation. Fondements, méthodes, pratiques. Paris : Téraèdre.

Delory-Momberger, C. (2009). La condition biographique. Essai sur le récit de soi dans la modernité avancée. Paris : Téraèdre.

Denzin, N. (1989). Interpretativ interactionnism. New-York : Sage Publications.

Ferenczy, F. (2006). Le traumatisme. Trad. Equipe du Coq du Héron. Paris : Payot \& Rivages, $1^{\circ}$ éd. 1927.

Hesbeen, W. (2018). Les mots « fragiles » du prendre soin. Santé mentale, 224/janv. 2018, 34-37.

Husserl, E. (2008). Méditations cartésiennes. Trad. E. Levinas \& M. Pfeiffer. Paris : Vrin, $1^{\circ}$ éd. 1929.

Janner Raimondi, M. (2018). Empathie du chercheur dans la conduite d'entretiens d'adolescents malades chroniques : nécessité d'un encadrement éthique et d'une posture clinique. In : M. Janner Raimondi \& A. Trouvé (Dir.). L'engagement éthique en éducation et en recherche. (Chapitre V, p. 93-113). Rouen : Presses universitaires Rouen Le Havre, collection « Penser les valeurs en éducation et en formation $»$.

Janner Raimondi, M. (2017). Visages de l'empathie en éducation. Nîmes : Champ Social.

Janner-Raimondi, M. (2015). Questions d'éthique et entretien : une approche philosophique. In : D. Bedoin \& R. Scelles (dir.) S'exprimer et se faire comprendre. Entretiens et situations de handicap. (p. 23-43). Toulouse : Erès, collection : Connaissance de la diversité ».

Lani-Bayle, M. \& Mallet, M-A. (2006). Evénement et formation de la personne. Paris : L'Harmattan. Laub, D. (2015). Le défaut d'empathie. Le Coq-héron, 220(1), 29-47. doi :10.3917/cohe.220.0029.

Lesourd, F. (2019). Événement. In C. Delory-Momberger (dir.). Vocabulaire des histoires de vie et de la recherche biographique. (pp. 80-81). Editions : Érès.

Lesourd, F. (2009). L'homme en transition. Education et tournants de vie. Paris : Anthropos.

Levinas, E. (1974). Autrement qu'être ou au-delà de l'essence. Paris : Livre de poche.

Lorelle, P. (2016). L'expérience traumatique : Emmanuel Levinas et Claude Romano. In : P. Cabestan (Coord.). L'événement et la raison autour de Claude Romano. (pp. 47-71). La Plaine Saint Denis : Isi Print

Maldiney, H. (1997). Penser l'homme et sa folie. Grenoble : Editions Jérôme Million

Marcelli, D. \& de La Borie, G. (2012). Tracas d'ados, soucis de parents. Paris : Albin Michel.

Marcelo, G. (2016). Claude Romano et la critique de la raison. In : P. Cabestan (Coord.). L'événement et la raison autour de Claude Romano. (pp. 167-194). La Plaine Saint Denis : Isi Print.

Marin, C. (2019). Rupture(s). Paris : Éditions de l'Observatoire.

Marion, J-L. (2010). Certitudes négatives. Paris : Grasset.

Merleau-Ponty, M. (1945). Phénoménologie de la perception. Paris : Gallimard.

Niewiadomski, C. \& de Villers, G. (Dirs. 2002). Souci et soin de soi. Liens et frontières entre histoire de vie, psychothérapie et psychanalyse. Paris : L'Harmattan. 
Rancière, J. (2000). Le partage du sensible. Paris : La Fabrique.

Rostaing, C. (2010). De la reconnaissance en prison? Vulnérabilité des ordres de reconnaissance et du mépris. La reconnaissance à l'épreuve. Explorations socio-anthropologiques. (pp. 141-148). Lille : Presses Universitaires du Septentrion.

Romano, C. (2010). Au cœur de la raison, la phénoménologie. Paris : Gallimard.

Romano, C. (1998). L'événement et le monde. Paris : PUF.

Sironi, F. (2004). Les mécanismes de destruction de l'autre. In : A. Berthoz (Dir.). L'empathie. (pp. 225-246). Paris : Odile Jacob.

Tisseron, S. (2014). L'empathie au cœur du jeu social. In: M. Botbal, N. Garret-Gloanec \& A. Besse (Dirs.). L'empathie au carrefour des Sciences et de la clinique. (pp. 193-202). Cerisy : Ed. Doin.

Vallee, M-A. (2016). Par-delà le principe de raison : la phénoménologie de l'événement chez JeanLuc Marion et Claude Romano. In : P. Cabestan (Cord.). L'événement et la raison. Autour de Claude Romano. (pp. 17-29). Paris : Le Cercle Herméneutique.

Van Broek, N. \& Rillaer, J. (2012). L'accompagnement psychologique de l'enfant malade. Paris : Odile Jacob.

de Villers, G. (2019). Sujet/Subjectivation. In: C. Delory-Momberger (dir.). Vocabulaire des histoires de vie et de la recherche biographique. (pp. 172-175). Editions : Érès.

de Villers, G. (2011). L'approche autobiographique : regards anthropologique et épistémologique et orientations méthodologiques. Recherches sociologiques et anthropologiques, 42-1, 2011, 25-44. https://doi.org/10.4000/rsa.653

Vincent-Buffaut, A. (2019). Délicatesse. In : A. Vandevelde-Rougale et alii (Dirs). Dictionnaire de sociologie clinique. (pp. 182-183). Toulouse : Erès.

Zanna, O. (2015). Savoir composer avec les contraintes pour se (re) éduquer : entre le scylla du laisser-faire et le charybde de la frustration. Le sociographe, 2015/5, Hors-Série, n 8, 85-103.

\section{NOTES}

1. Notamment, les parents d'enfants avec handicap, rencontrés dans le cadre de projets de recherche financés : "L'accueil des jeunes enfants en situation de handicap dans des dispositifs dits " ordinaires » : crèche et école maternelle » (2009-2012) U. Rouen ; "Petite enfance et handicap : accueil collectif et coordination institutionnelle » (2013-2015) U. Rouen, dir. D. Bedoin, M. Janner Raimondi, N. Guirimand.

2. Participation à trois projets de recherche financés: «Les adolescent.e.s face à la maladie chronique : à la croisée de la philosophie-phénoménologie, de la médecine et des sciences de l'éducation » (ADOCHRONIQ)»(2015-2019) dir. N. Depraz, U. Rouen ; "E-Plateform Innov' Maladies rares»(2015-2018), dir. D. Farge-Bancel, C. Tourette-Turgis, U. Paris 6, C. DeloryMomberger, U. Paris 13 et Association Française du Lupus et autres maladies auto-immunes; "Croiser les expériences: vers une transformation de l'action de soin dans la maladie chronique » (2017-2020), dir. G ; Beck-Wirth, PH au CHG Mulhouse, garant scientifique M. Janner Raimondi.

3. Participation au projet de recherche financé : « La vie au quotidien des personnes en Etat Végétatif Chronique (EVC) ou en Etat Pauci Relationnel (EPR)» (2016-2020) dir. A. Boissel, U. Rouen et N. ProiaLelouey, U. Caen. 


\section{AUTEUR}

\section{MARTINE JANNER RAIMONDI}

Professeur en Sciences de l'éducation, Université Sorbonne Paris Nord, EXPERICE 